${ }^{1}$ Department of Anesthesiology and Intensive Care Medicine, Medical University Hospital Innsbruck, Innsbruck, Austria, ${ }^{2}$ Austrian Board of Alpine Safety, Innsbruck, Austria, ${ }^{3}$ Alpine Police, Federal Ministry of the Interior, Vienna, Austria, ${ }^{4}$ Barts Heart Centre, St. Bartholomew's Hospital, Queen Mary University of London, London, UK

Introduction.-Since November 1, 2005, the Austrian Alpine Police records data for every mountain accident, which is reported to an emergency dispatch center, in a digital database. Still, no medical data on lightning injuries in the Austrian mountains are available.

Objective.-To analyze lightning casualties in the Austrian mountains with respect to accident mechanism, injury pattern, and medical outcome.

Methods. - The database was evaluated regarding emergencies caused by lightning that occurred from November 1, 2005, to December 31, 2014. Additionally, data for lightning strike victims who were treated at the Innsbruck University Hospital in Western Austria were included. This tertiary level hospital in the Eastern European Alps has a catchment area of approximately $25,000 \mathrm{~km}^{2}$ and 3 million inhabitants. Data included sex, age, victim's activity at the time of the injury, severity of injury, and time and location of the accident.

Results.-In the study period, 67,807 alpine accidents were registered in the database, and 70,675 casualties were involved. The search for lightning casualties revealed 96 persons. Sixty-four of these fulfilled inclusion criteria for this study. Sixty of the victims (94\%) survived the lightning accident. Four people died; among these were 2 hunters who were killed while in a high seat and a ranger who was felling trees. The fourth person was a hiker who was hiding from the thunderstorm below a mountain peak when he was hit.

Conclusions.-Most lightning accidents happen in late afternoon in summer. Climbers, mountaineers, and hikers are most commonly hit; however, they also make up the highest share of people who are active in the mountains. People next to trees seem to be at high risk of being struck by lethal lightning. Lightning accidents affecting humans should be better interlinked with hospital data to gain better insight into accident mechanism and outcome.

Trauma Training for Journalists: Developing and Implementing an Intercultural Medical Curriculum for Journalists Working in Regions of Conflict

David S. Young, MD, MS ${ }^{1,2}$; Michael Puntis, MB, BChir $^{3}$

${ }^{1}$ University of Colorado Denver School of Medicine, Aurora, CO, USA,

${ }^{3}$ Rush University Medical Center, Chicago, IL, USA, ${ }^{2}$ St. George's

University, London, UK

Introduction.-Almost 1200 journalists have died on assignment since 1992. Most deaths resulted from trauma (automobile accidents, gunfire) and occurred in the Middle East, Africa, and Latin America. While deaths of highprofile Western reporters have brought attention to inherent risks of reporting, $88 \%$ of deaths were local journalists earning a meager wage. In recognition of these dangers, major news agencies have endorsed freelance protection standards including basic medical training. However, trainings typically occur in North America and Western Europe-never reaching those most at risk.

Objective.-To establish and evaluate a culture-neutral course, to be delivered with equal effectiveness in a number of geographical areas, that will educate journalists on the basics of medical care in remote and resource-limited locations.

Methods.-A multinational team of physicians and paramedics was formed and led by a retired professional journalist. The team developed the curriculum based on wilderness medicine and combat experience. The course was administered in Beirut, chosen for its proximity to conflict and large media outlets. At the completion, students were tested on content and provided feedback.

Results.-Seventeen students of various nationalities (Lebanese, American, British, French, Canadian, Egyptian, Syrian, and Spanish), started and completed the course. Those speaking primarily English scored $80 \%$ on average compared with $71 \%$ for those speaking English secondarily and 60\% for Arabic-speaking students.

Conclusions.-Developing and implementing a culture-neutral course proved to be a lengthy and challenging process. Live interpreters were invaluable for Arabic-speaking students, in addition to eliminating medical jargon and focusing on active demonstrations and scenarios. Despite this, language barriers contributed to decreased retention of approximately one-quarter of the lecture material. Special attention to sex-norms in an Arabic country was necessary when undergoing scenario training. Lessons learned in the process will be applied to future TTJ courses and shared with those developing similar curricula.

Telesound: Can Real-Time Video Streaming of Ultrasound Imaging from Remote Locations Yield An Accurate Diagnosis?

Rachel E. Whitney; Antonio Riera; Lei Chen

Yale University, New Haven, CT, USA

Background.-Ultrasound has been proven to help medical decision-making in remote locations. Use of cell phones in medical care has become a popular trend given their availability, and $85 \%$ of the world has access to a 3G network.

Objective.-To evaluate live-stream video for interpretation of ultrasound using a $3 \mathrm{G}$ network.

Methods. - We evaluated the inter-rater reliability of assessment of ultrasound scans for abdominal free fluid, intussusception, and hip effusions. Deidentified 6-second video clips in .mp4 format were downloaded to a laptop: 58 focused assessment with sonography in trauma (FAST) scans, 44 scans evaluating for intussusception, and 28 scans evaluating for hip effusions. A bedside ultrasound expert evaluated each scan and marked it positive or negative for pathology. Using an iPhone 3G for remote "telesound" consultation, we used Skype to connect to our second ultrasound expert located in Ethiopia, who repeated the process over a live-stream video connection. When the remote expert returned from Ethiopia, evaluation of the scans was repeated as a bedside reviewer. Inter-rater reliability analysis using the Kappa statistic and percent agreement were performed to determine consistency among raters.

Results. - The Kappa values between the bedside and remote evaluators for FAST, intussusception, and hip effusion were 0.748 (95\% CI 0.576-0.92), 0.816 (95\% CI $0.648-0.984)$, and 0.764 (95\% CI 0.519-1.0), respectively. The percent agreements after adjusting for chance were $86 \%, 80 \%$, and $88 \%$, respectively. The Kappa values between the bedside assessments by each evaluator were 0.851 (95\% CI $0.71-0.992)$, 0.8 (95\% CI 0.616-0.984), and 0.747 ( $95 \%$ CI $0.479-0.884$ ), respectively. The percent agreements after adjusting for chance were $92 \%, 81 \%$, and $88 \%$, respectively.

Conclusion.-Live-stream video conference using 2 cellphones over a 3G network is a feasible and accurate method for consultation of ultrasound scans from a remote location.

\section{CPR in Whitewater Drowning Victims: Is It Possible To Perform Adequate Chest Compressions Through a Personal Flotation Device (PFD)?}

Scott McCorvey ${ }^{1}$; Erin Lurie $^{2}$; Robert D’Zio ${ }^{2}$; Michael Caudell ${ }^{2}$ ${ }^{1}$ Maine Medical Center, Portland, ME, USA, ${ }^{2}$ Medical College of Georgia at Augusta University, Augusta, GA, USA

Introduction.-Whitewater activities such as rafting, canoeing, and kayaking are growing in popularity throughout the country. More than 17 million Americans participated in these activities in 2008 alone. With this increase in participation has come an increase in paddling-related fatalities. It is estimated that $25 \%$ to $30 \%$ of all deaths are attributable to cardiac arrest.

Objective.-To examine the effects of a personal flotation device (PFD) on cardiopulmonary resuscitation (CPR) chest compression depth.

Methods. - This is a prospective study, using volunteers certified in CPR by the American Heart Association (AHA) and the Laerdal Medical Simulation Mannequin (SimMan) to evaluate the efficacy of chest compressions while wearing 3 different PFD styles. Compression depth, rate, and variance were recorded during 2-minute cycles of chest compressions and averaged for each test group. Statistical analysis was performed using a student's t-test.

Results. - Data were compared between the control group and 3 different PFD styles measuring compression depth and rate ( $\mathrm{n}=37$ per group). All groups had adequate rates of compression. The mannequin without a PFD (control) demonstrated an average depth of $49.5 \mathrm{~mm}$. The 3 PFD styles allowed an average compression depth of $48.7 \mathrm{~mm}(P=.61), 45.7 \mathrm{~mm}(P<.016)$, and $45.2 \mathrm{~mm}(P<.019)$.

Conclusion.-2015 AHA guidelines recommend adult CPR compression depth of 50 to $60 \mathrm{~mm}$. Other studies have shown maximum survival at lower 
compression depth ranges (peak $45.6 \mathrm{~mm}$ ). Optimal CPR efforts may be achievable in suspected drowning victims while wearing a PFD, but did not reach current AHA compression depth guidelines. While there was a statistically significant difference in compression depth achievable among the 3 varieties of PFDs, its clinical significance (3-4 mm in depth) is uncertain. Further studies are needed to test other PFDs, in particular those that are used in the commercial rafting industry.

\section{Low-Cost, Highly Adaptable Mannequin for Use in Wilderness Medicine Simulation}

Joshua Nichols; Stephanie Lareau

Virginia Tech Carilion Clinic, Roanoke, VA, USA

Introduction.-Simulation-based learning is an effective means of teaching disease management and procedural skills for treating critically ill patients in the austere environment. Although high fidelity mannequins can simulate multiple disease states and procedures, it is usually cost prohibitive to use such expensive equipment in the wilderness. It is therefore common practice for simulations to involve a "live actor" patient combined with standalone anatomical models for conducting procedures. This arrangement has certain limitations, including putting the live actor at risk as well as inability to simulate certain disease states. We seek to understand the feasibility of creating a low-cost mannequin platform that can be adapted to simulate various disease states and procedural skills.

Objective.-To determine the feasibility of creating a simulation mannequin for less than $\$ 500$ that can simulate heart and lung sounds, cricothyrotomy, tension pneumothorax needle decompression, and arterial injury.

Methods.-A polystyrene adult male mannequin (\$115) was purchased from AMT Mannequins. This mannequin then was modified to allow for simulated procedures. A Bluetooth speaker $(\$ 20)$ was mounted in the chest that could play heart and lung sounds. The anterior neck was removed and a cricothyrotomy simulator was constructed in its place using foam tape, gauze pads, ketchup packets, and ventilator tubing. A tension pneumothorax needle decompression simulator was placed in the right midclavicular line using foam tape, gauze, and InstaMorph moldable plastic (\$35), which was used to construct custom ribs. A femoral arterial injury was simulated using a $1 \mathrm{~L}$ bag of normal saline in a pressure bag mounted inside the torso, connected to IV tubing running into the thigh.

Results. - The total cost of the custom mannequin was approximately $\$ 170$.

Conclusions.- It is feasible to construct an inexpensive simulation mannequin that can be used to simulate heart and lung sounds and for practicing multiple procedural skills.

\section{Which Matters More for Backcountry Preparedness: Wilderness or Medical Training?}

Michael D. Yue ${ }^{1}$; David W. Spivey ${ }^{1}$; Daniel B. Gingold ${ }^{1}$; Douglas G. Sward ${ }^{1,2}$

${ }^{1}$ Department of Emergency Medicine, University of Maryland School of Medicine, Baltimore, MD, USA, ${ }^{2}$ Program in Trauma, University of Maryland School of Medicine, Baltimore, MD, USA

Objective.-To identify how previous medical and wilderness training correlate to levels of preparedness for acute mountain sickness (AMS) as well as illness and injury in backcountry hikers. Few previous studies have directly assessed preparedness in the backcountry.

Methods.-We conducted a cross-sectional, convenience survey on trails in 4 sections of the Rocky Mountain National Park during 2 weeks in July and August 2015. The study group consisted of 379 hikers in the backcountry who consented to a written survey that assessed demographics, wilderness experience, altitude experience, hiking equipment, communications devices, and pretrip planning

Results. - Factors including wilderness training (WFA/WFR/WEMT), wilderness experience, and altitude experience all affected hiker preparedness. Respondents with medical training were more prepared for injury and illness in the backcountry compared with those without medical training, $(37.7 \%$ vs $20.7 \%, \mathrm{p}<.001)$. They were also more prepared for AMS $(62.3 \%$ vs $34.3 \%$, $\mathrm{p}<.001$ ). Respondents with wilderness training (WFA, WFR, and WEMT) were not significantly more likely to be prepared for illness and injury (31.8\% vs
$22.0 \%, \mathrm{p}<.11)$. However, they were more likely to be prepared for AMS $(52.3 \%$ vs $36.8 \%, \mathrm{p}=.025)$. Adjusting for experience, wilderness training, age, and gender, we found that medical training was associated with increased preparedness for injury and illness in the backcountry (2.71 Odds Ratio, 95\% CI 1.5-4.89) and AMS (2.72 Odds Ratio, 95\% CI 1.51-4.91).

Conclusions.-Medically trained and wilderness-trained individuals are more likely to be prepared for altitude and medical trauma than their nontrained counterparts. While it is neither practical nor feasible to train most hikers to be emergency medical technicians, nurses, or physicians, our data suggest that wilderness training is helpful in mitigating risk in the backcountry.

\section{Severe Hypertension and Efficacy of Antihyperten- sive Medications at High Altitude}

T. Douglas Sallade ${ }^{1}$; Linda E. Keyes ${ }^{2,3}$; Alison Sheets ${ }^{3}$; Jennifer Starling ${ }^{2,4}$; Sushil Pant ${ }^{5,6}$; David Young ${ }^{2}$; David Twillman ${ }^{2}$; Nirajan Regmi ${ }^{5}$; Benoit Phelan ${ }^{7}$; Purshotam Paudel ${ }^{5,8}$; Matthew McElwee ${ }^{9}$; Theodore McConnell ${ }^{10}$; Luke Mather ${ }^{11,12}$; Charles Duke ${ }^{13}$; Devlin Cole ${ }^{9}$; Buddha Basnyat ${ }^{14,15}$

${ }^{1}$ Philadelphia College of Osteopathic Medicine, Philadelphia, PA, USA, ${ }^{2}$ Department of Emergency Medicine University of Colorado, Aurora, CO, USA, ${ }^{3}$ Longmont United Hospital, Longmont, CO, USA, ${ }^{4}$ Colorado Permanente Medical Group, Saint Joseph Hospital, Denver, CO, USA, ${ }^{5}$ Mountain Medicine Society of Nepal, Kathmandu, Nepal, ${ }^{6}$ Khunde Hospital, Kathmandu, Nepal, ${ }^{7}$ Integrated Family MedicineEmergency Medicine Residency, Saint John Regional Hospital, Dalhousie University, Halifax, NS, Canada, ${ }^{8}$ District Hospital, Dhading, Nepal, ${ }^{9}$ Case Western Reserve University School of Medicine, Cleveland, OH, USA, ${ }^{10}$ McGill University, Montreal, QC, Canada, ${ }^{11}$ Family Medicine Residency of Idaho, Boise, ID, USA, ${ }^{12}$ University of Washington School of Medicine, Seattle, WA, USA, ${ }^{13}$ University of Tennessee Health Science Center, College of Medicine, Memphis, TN, USA, ${ }^{14}$ International Society of Mountain Medicine, Montreal, QC, Canada, ${ }^{15}$ Oxford University Clinical Research Unit-Nepal, Mountain Medicine Society of Nepal, Himalayan Rescue Association, Kathmandu, Nepal

Background.-Severe and uncontrolled hypertension have been reported in high altitude sojourners but have not been studied systematically. Additionally, few studies have evaluated the efficacy of antihypertensive medications at high altitude. We documented the prevalence of severe hypertension and efficacy of antihypertensive medications in high-altitude trekkers in Nepal.

Methods.-Observational cohort study in Nepal's Solukhumbu Valley. We recruited trekkers 18 years of age and older. Subjects reported demographics, medical history, and medications. Resting blood pressure (BP) was recorded at $2860 \mathrm{~m}, 3400 \mathrm{~m}$, and $4300 \mathrm{~m}$ on ascent and descent. Severe hypertension was defined as systolic BP $\geq 180$ and/or diastolic BP $\geq 100$.

Results.-We enrolled 60 self-reported hypertensive (HTN) trekkers and 606 normotensive (NTN) trekkers. Out of 2158 BP measurements at all altitudes, $109(5 \%)$ were severe. Of these, 39 occurred in $22(37 \%)$ HTN trekkers and 70 were distributed among $51(8 \%)$ NTN trekkers. Occurrence of severe hypertension was similar across altitudes $(P<.05)$. No subject was symptomatic. Among HTN trekkers, 10 took no antihypertensives and 5 of those had one or more severe BP measurements, accounting for $21 \%$ of all severe BPs in HTN trekkers. Seven out of 14 subjects on angiotensin converting enzyme inhibitors (ACEI) accounted for another one-third of all severe BPs. No subject taking a beta-blocker (BB), thiazide, ACEI + calcium channel blocker (CCB), ACEI + BB, or angiotensin receptor blocker (ARB) + alpha-1 antagonist had severe hypertension. Severe hypertension occurred in a smaller proportion of trekkers on combinations of $\mathrm{CCB}$ plus either ACEIs (0/3) or ARBs (1/3), and those taking more than 3 antihypertensives $(1 / 3)$

Conclusions.-Asymptomatic severe hypertension occurred at high altitude in normotensive and hypertensive subjects, but was more common in those with underlying hypertension. The clinical importance of these episodes is 